\title{
Prognostic Values of Galectin-3 and the Macrophage Migration Inhibitory Factor (MIF) in Human Colorectal Cancers
}

Hugues Legendre, M.D., Christine Decaestecker, Ph.D., Nathalie Nagy, M.D., Alain Hendlisz, M.D., Max-Peter Schüring, Ph.D., Isabelle Salmon, M.D., Hans-Joachim Gabius, Ph.D., Jean-Claude Pector, M.D., Robert Kiss, Ph.D.

Divisions of Digestive Surgery (HL, J-CP) and Gastroenterology (AH), Institut Bordet; Laboratory of Histopathology (CD, RK), Faculty of Medicine; Laboratory of Pathology (NN, IS), Erasmus University Hospital; and Université Libre de Bruxelles, Brussels, Belgium; and Institute of Physiological Chemistry (M-PS, H-JG), Faculty of Veterinary Medicine, Ludwig-Maximilians-University, Munich, Germany

This study aims to investigate whether the immunohistochemical levels of expression of galectin-3 and the macrophage migration inhibitory factor (MIF) are associated with prognostic values in human colorectal tumors. This was performed on 99 specimens including 69 colorectal tumors (17 Dukes A, 19 Dukes B, 15 Dukes C and 18 metastatic tumors that we labeled as $D), 10$ hepatic metastases from colorectal cancers and 20 normal specimens (biopsies). The immunohistochemical levels of expression of MIF and galectin-3 were quantified on routine histological slides by means of computerassisted microscopy. Separate analyses were performed on epithelial and connective tissue. The levels of expression of both MIF and galectin-3 were very significantly higher in epithelial tumor tissue when compared with normal epithelial specimens. A positive and significant correlation between MIF and galectin-3 expression was evidenced in connective tumor tissue, and in particular in the cases associated with short survival periods ( less than 5 years). In the case of the Dukes A or B tumors, we established two new prognostic groups (labeled I and II) on the basis of the levels of galectin-3 expression measured in the tumor epithelium. In the case of the Dukes $C$ or D tumors, we established two other prognostic groups (labeled III and IV) on the basis of the levels of MIF expression measured in

Copyright (C) 2003 by The United States and Canadian Academy of Pathology, Inc.

VOL. 16, NO. 5, P. 491, 2003 Printed in the U.S.A

Date of acceptance: February 21, 2003.

Christine Decaestecker is a Research Associate and Robert Kiss a Director of Research with the "Fonds National de la Recherche Scientifique" (FNRS, Belgium).

Address reprint requests to: Robert Kiss, Ph.D., Laboratory of Histopathology, Faculty of Medicine, Université Libre de Bruxelles, 808 route de Lennik, 1070 Brussels, Belgium; fax: 322-555-6285; e-mail: rkiss@ulb.ac.be.

DOI: 10.1097/01.MP.0000068235.45178.C the connective tissue. Kaplan-Meyer analyses confirmed the additional prognostic values (as compared with conventional clinical staging) given by this new classification (groups I to IV). They show that the Dukes A or B tumors characterized by low levels of galectin-3 expression in the tumor epithelium are associated with significantly better prognoses than those characterized by high levels. In addition, the Dukes C or D tumors characterized by high levels of MIF expression in the connective tumor tissue are associated with significantly better prognoses than those characterized by low levels. In conclusions, MIF and galectin-3 expression levels in colorectal tumors are related to their levels of biological aggressiveness. These markers could be used to identify patients at risk, for whom more aggressive adjuvant therapy seems to be indicated.

KEY WORDS: Colorectal cancer, Connective tissue, Dukes classification, Epithelium, Galectin-3, MIF, Prognosis.

Mod Pathol 2003;16(5):491-504

In the industrial world colorectal cancer is the second most common form of cancer, with some 140,000 new cases diagnosed annually (1-4). Forty percent of these patients die due to the progression of their colorectal cancers, even if about $75 \%$ of these patients have undergone surgery for therapeutic purposes (1-4). Adjuvant therapies (chemotherapy, radiotherapy) can be performed in relation with tumor prognosis in addition to surgery (5). Prognostic evaluations are established based on distinct criteria including the macroscopic appearance of the colon (the presence or absence, for example, of occlusion or perforation, etc.), the histological tumor type, the degree of tumor cell differentiation and the presence of vascular and/or 
lymphatic emboli (6-8). The first system of predicting the progression of colorectal cancer was proposed by Dukes in 1932 (9). This classification has proven its value and remains in use today either in its original or in one of its modified forms. The different ways (clinical staging, histopathological grading, measures of biological markers in the sera, etc.) of classifying human colorectal cancers in terms of potential biological aggressiveness in fact enable prognostic assessments to be provided for particular clinical groups of patients, but not for individual patients $(10,11)$. Thus, in addition to conventional methods of classification, the search for biological factors as new prognosticators remains of major interest. The Vogelstein group specified the sequence of genomic mutations leading to the transformation of normal colorectal mucosa into adenomas and, subsequently, into cancers (1214). This means that when colorectal tissue accumulated an increasing number of different mutations, it can be assigned to a certain position in the normal-adenoma-cancer sequence. However, this pinpointing will not enable precise prognoses to be established at individual level. The identification of certain gene products (proteins including their posttranscriptional modifications) whose expression levels are significantly down- or upregulated in cancers as compared with benign tissue can be used for such purposes. In this context, the analysis of protein-carbohydrate interactions is receiving increasing attention. Because it is known that malignancy is associated with characteristic changes in glycosylation, the ensuing alterations in this system (sugar coding) can well have a bearing on establishing the tumor phenotype (15-19). One family of proteins of interest in this respect are the galectins, an evolutionarily ancient group of lectins that are found in species ranging from sponges and nematodes to humans (20-24). These $\beta$-galactosidespecific lectins are able to mediate cell adhesion, to regulate cell growth, and to trigger or inhibit apoptosis: they are therefore suspected of playing a number of important roles in tumor development (20-24). Galectin-3, one of the 14 types of mammalian galectins identified to date, has already been shown to be associated with colon cancer development: it is frequently expressed by various colon cancer cell lines $(25,26)$ and seems to be more highly expressed in colon cancer cells with increased metastatic capacity (27). Galectin-3 ligands include, for example, carcinoembryonic antigen (25) and colon cancer mucin (27), which are already known to be involved in colon cancer progression $(25,27)$. However, as emphasized by Itzkowitz (28), an inconsistent picture had emerged from the literature with respect to the prognostic value of galectin-3 in human colon cancer. Whereas some studies report decreasing levels of galectin-3 ex- pression in colon carcinomas $(29,30)$, others describe increasing galectin-3 expression during colon cancer progression (31-33). In the present study we therefore investigated whether galectin-3 expression differs in normal colon tissue, primary colorectal cancers and colorectal cancer metastases. We then investigated whether galectin-3 expression differs in primary colorectal cancers depending on their Dukes' clinical stages. Finally, we investigated whether galectin-3 expression differs in a particular Dukes' clinical stage in relation to patients' survival. Immunohistochemical galectin-3 expression was quantitatively monitored separately in epithelial and connective tissue.

In addition to galectin-3, we also investigated the potential prognostic value of the macrophage migration inhibitory factor (MIF) for the following reasons. Firstly, we had observed that MIF expression correlates with the expression of galectin-3 in relation to recurrences in human cholesteatomas (34). Secondly, recent studies suggest that MIF, first identified nearly 40 years ago as a potent proinflammatory cytokine, may also contribute to the multiple aspects of tumor progression and neoplasia including impairment of p53 activity (35-37). Thirdly, MIF activity has already been shown to differ in normal and neoplastic colon tissue $(38,39)$. We thus characterized the MIF expression (using a polyclonal antibody) in the present study exactly as we did for galectin-3.

\section{MATERIALS AND METHODS}

\section{Clinical Data}

We collected for immunohistochemical analysis 69 colorectal tumors, 10 hepatic metastases from colorectal cancers and 20 normal specimens. The normal cases came from routine biopsies made on colorectal areas without any sign of clinical aberrations and carried out on patients subjected to total colonoscopy for screening purposes. The cancer series includes a retrospective, continuous, unselected cohort of patients who underwent a primary resection with curative intent. Cancer staging was performed according to the three categories of the Dukes' classification (Dukes A, B and C) (9). At least 12 lymph nodes were analyzed per case to assess the Dukes stage. We added to this series a set of tumors resected from patients with distant metastases at the time of diagnosis. These tumors were classified in category "D." Finally, the 69 cancers included 17 Dukes A, 19 Dukes B, 15 Dukes C and 18 D. Of these, 19 were rectal tumors (8 Dukes A, 6 $\mathrm{B}, 4 \mathrm{C}$ and $1 \mathrm{D}$ ). The ages of the 79 patients (with colorectal tumors or hepatic metastases) ranged from 29 to 88 years (mean $=64$ ) and did not significantly vary between the groups of patients as- 
sociated with the different tumor stages. There were 40 men and 39 women. All the patients were operated on by two surgeons (HL and J-CP) at the Institut Bordet (Brussels, Belgium). All those suffering from Dukes $C$ tumors received adjuvant chemotherapy with levamisole and fluorouracil following the so-called "Mayo Clinic regimen" (40). Patients with distant metastasis at the time of diagnosis (stage D) were treated following protocols undergoing at the time of diagnosis (the association of FU and CPT11 or oxaliplatin). The clinical follow-ups of all the colorectal cancer patients under study are illustrated in the Results. All the specimens were first fixed in buffered formalin before further processing for paraffin embedding. Tissue samples from 4 cases were also kept in liquid nitrogen for Western blotting and PCR analysis.

\section{MIF and Galectin-3 Immunohistochemistry}

All the histochemical procedures were carried out as detailed previously $(34,41)$. Briefly, $5 \mu \mathrm{m}$-thick sections were taken from each of the 99 clinical samples (i.e., 20 normal cases, 69 colorectal tumors and 10 hepatic metastases). Incubation with the antibody-containing solutions was carried out at 25 $\pm 1^{\circ} \mathrm{C}$ for 60 minutes at a concentration of $10 \mu \mathrm{g} /$ $\mathrm{mL}$. The extent of the specifically bound antibodies was visualized by avidin-biotin-peroxidase complex (ABC) kit reagents (Vector Labs, Burlingame, CA), with diaminobenzidine $/ \mathrm{H}_{2} \mathrm{O}_{2}$ as the chromogenic substrates.

The anti-MIF antibody is a polyclonal rabbit antibody raised against MIF purified from bovine lenses following chromatographical steps (42) and high-resolution preparative gel electrophoresis. The antibody was also assessed for specificity by Western blotting, as described below. The polyclonal antibody against galectin-3 produced by recombinant expression with the expression vector prCBP35, kindly provided by Dr. J.L. Wang (East Lansing, MI) was raised as described previously (41). To exclude cross-reactivity to galectin family members present in colorectal cancer tissue, Western blotting with purified galectins- $1,-4,-7$, and -8 were performed in addition to routine quality controls. These controls included the omission of the incubation step with polyclonal antibody to exclude any kit-reagent-independent staining (e.g., by binding of the $\mathrm{N}$-glycan chains of the kit reagent glycoproteins avidin and/or horseradish peroxidase to glycanbinding sites in the tissue or of avidin to endogenous biotin), the incubation with serum containing no specific antibody to exclude antigen-dependent binding via Fc receptors, the immunohistochemical analysis of cases shown to be negative/positive in analysis by Western blotting (for technical details, see below) and the preincubation of the polyclonal antibodies with an excess of antigen to saturate their antigendependent binding in situ.

\section{Computer-Assisted Microscopy}

The immunohistochemical galectin-3 and MIF stainings were quantitatively characterized in two different ways by a SAMBA 2005 computer-assisted microscope system (Samba Technologies, Grenoble, France) with a 20x (aperture 0.50) magnification lens. While the Labeling Index (LI) refers to the percentage of tissue area specifically stained by a given histochemical marker, the Mean Optical Density (MOD) denotes the mean staining intensity computed in immunopositive cells only. The way in which we used the computer-assisted system to quantify the histochemical staining is detailed elsewhere, as are all the standardization procedures dealing with the manner in which we used the computer-assisted microscopy and ensuring measure reproducibility $(34,41,43)$. A negative histological control slide (from which either the primary anti-MIF or the anti-galectin-3 antibody was omitted, as mentioned above) was analyzed for each case under study. The software used in the computer-assisted microscope automatically subtracted the LI and MOD values of the negative control sample from each corresponding positive one.

Two types of histological structure were analyzed for each of the cases under study. The first of these structures was the epithelial component while the second was the adjacent peritumoral connective tissue. The software that we set up $(34,41,43)$ enabled these two tissues to be analyzed separately in each case by means of a computer-mouse linked to this software. This mouse enabled the epithelial component and the connective tissue to be delineated on a control video screen, so leading to a separate quantitative analysis of each of the biological markers under study (as illustrated in Fig. 1, G-I). Ten fields of between 60,000 and 120,000 $\mu \mathrm{m}^{2}$ each were scanned for epithelial and connective tissue in each of the 99 cases studied.

\section{Western Blotting Analyses}

The check for anti-galectin-3 and anti-MIF antibody specificities was carried out on four human colon cancers (one for each Dukes stage, with the addition of "Dukes D") and two positive controls. These positive controls included two human cell lines (HeLa [a human cervical carcinoma cell line] and LoVo [a human colon cancer cell line] obtained from the American Type Culture Collection; Manassas, VA).

Western blotting analyses were carried out as detailed previously (44). Briefly, after the colon tumor tissue and the cell lines had been homogenized, the tissue extracts were prepared by sonica- 

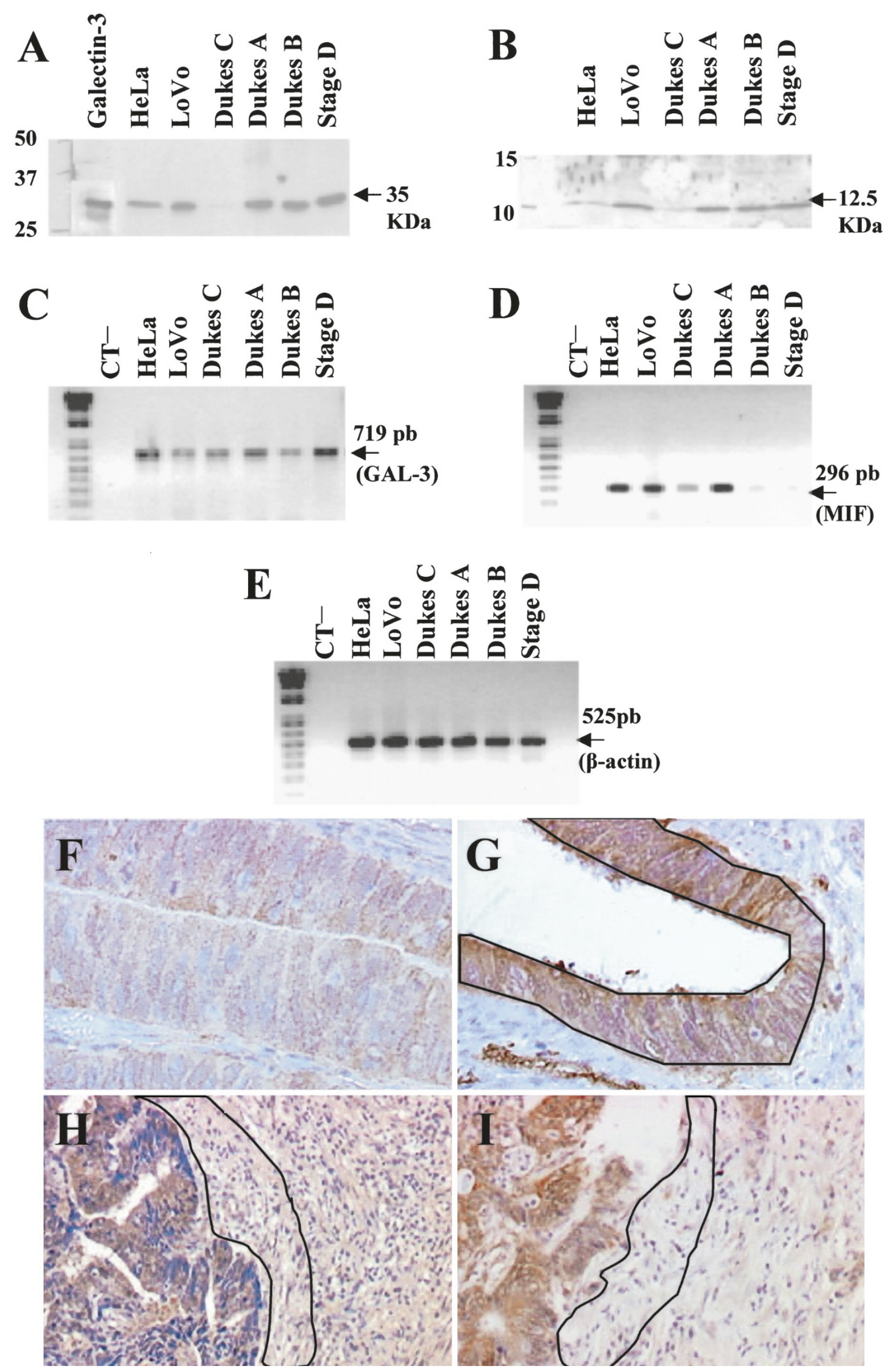
tion in PBS containing $1 \mathrm{~mm}$ PMSF and $10 \mu \mathrm{g} / \mathrm{mL}$ aprotinin. The protein concentration was determined by the BCA protein assay (Pierce, Polylabo, Antwerp, Belgium). The samples in each lane (10 $\mu \mathrm{g}$ for galectin-3 and $10 \mu \mathrm{g}$ for MIF) were loaded onto a $15 \%$ polyacrylamide gel under denaturing and reductive conditions. After electrophoresis, the proteins were transferred onto a Polyscreen ${ }^{\circledR P V D F}$ membrane (NEN ${ }^{\mathrm{TM}}$ Life Science Products, Boston, MA) by tank blotting. The target proteins (galectin-3 and MIF) were immunodetected by the affinity-purified antibodies $(0.05 \mu \mathrm{g} / \mathrm{mL}$ antigalectin-3 and $0.05 \mu \mathrm{g} / \mathrm{mL}$ anti-MIF) in T-TBS containing $5 \%$ of milk powder, in conjunction with a secondary antibody conjugated with horseradish peroxidase $(0.2 \mu \mathrm{g} / \mathrm{mL}$; NEN). The visualization of the immunoreactive proteins was carried out with reagents from a chemilunescence kit (NEL103, NEN). The control experiments included the omission of the incubation step with the specific antiprotein antibody (negative control). Fig. 1A and B illustrates the Western blots obtained for the antigalectin-3 and anti-MIF antibodies.

\section{Analysis of Galectin-3 and MIF RNA Expression in Human Colon Cancer Tissue}

Specimens from the same four human colon cancers and the two cell lines employed for Western blotting were lysed by adding Tripure ${ }^{\mathrm{TM}}$ Isolation Reagent (Roche, Mannheim, Germany). Total RNA was prepared according to the manufacturer's recommendation. Before cDNA synthesis, the RNA was incubated with DnaseI (1,71 IU/ $\mu \mathrm{l})$ (Roche Diagnostics) for $15 \mathrm{~min}$ at $37^{\circ} \mathrm{C}$. The RNA was purified by phenol/ chloroform extraction. One $\mu \mathrm{g}$ of total RNA was used as template for cDNA synthesis: reverse transcription was performed for $50 \mathrm{~min}$ at $42^{\circ} \mathrm{C}$ in RT buffer $(250$ mм Tris-HCl pH 8.3, $375 \mathrm{~mm} \mathrm{KCl,} 15 \mathrm{~mm} \mathrm{MgCl}_{2}, 10$ mм DTT, Gibco), oligodT (12-18) primers (25 ng/ $\mu$ l, Gibco), 500 nм dNTP, 8 IU RNAsin Ribonuclease Inhibitor (Promega, Leiden, The Netherlands) and 200 IU SuperscriptTM Rnase H Reverse Transcriptase (Gibco). The reaction was terminated by incubation for 15 minutes at $70^{\circ} \mathrm{C}$.

The integrity of the cDNA was confirmed by $\beta$-actin specific PCR analysis.

The primers for the galectin-3 and MIF were designed using the HYBsimulator software (Advanced
Gene Computing Technologies, Irvine, CA) (45) and purchased from Biosource Europe S.A. (Nivelles, Belgium). They were:

Galectin-3:

atggcagacaatttttcgctcc (sense), and atgtczccagaaattcccagtt (anti-sense); MIF:

gatgttcatcgtaaacaccaa (sense), and cgtaatagttgatgtagaccctg (anti-sense).

The PCR products were analyzed by electrophoresis on $1 \%$ agarose gel. Fig. 1, C-E illustrates the data obtained with the present PCR analyses.

\section{Data Analysis}

To compare independent groups of numerical data we used the non-parametric Mann-Whitney test (for independent samples) or the Wilcoxon matched pair tests (for paired data) because the conditions for applying the parametric $t$ tests were not satisfied in general. This is why data presentation uses the medians and the $25 \%$ and $75 \%$ percentiles. The rank correlation test involved the nonparametric Kendall test. Survival time analyses were performed using Kaplan-Meier curves and the log-rank test. As previously detailed $(46,47)$, we used a decision tree-based technique to determine threshold values (for the quantitative variables analyzed) able to discriminate between two different groups of patients. Briefly, this technique investigates all possible splits (for each variable) to find the split producing the largest improvement in discriminating groups of cases a priori defined. Finally, standard Cox regression analysis was used to fit to the survival data the explanatory models generated on the basis of different variables analyzed in the study. This enabled the possible simultaneous influence of several variables on the survival period to be tested. All the statistical analyses were carried out using Statistica (Statsoft, Tulsa, OK, USA).

\section{RESULTS}

Morphological Illustrations of Immunohistochemical Detection of Galectin-3 and MIF Expression in Human Colorectal Tissue

While Fig. 1, A-B illustrates the specificity check for anti-galectin-3 and anti-MIF antibodies per-

FIGURE 1. Panels A and B illustrate the specificity check for anti-galectin-3 (A) and anti-MIF (B) antibodies performed by means of Western blotting. Panels C-E show the check on the presence of galectin-3 (C) and MIF mRNAs (D), and cDNA quality illustrated on the $\beta$-actin mRNAs (E). These data are given for two cell lines (Hela and LoVo) used as positive controls and four human colon cancers (with different stages).Panels F-G $(\times 200)$ illustrate the immunostaining pattern for galectin-3 in a Dukes A colon cancer associated with a favorable prognosis (more than 8 years of survival postdiagnosis) and in a Dukes B colon cancer associated with a dismal prognosis (2 years of survival postdiagnosis), respectively. Panels H-I $(\times 100)$ show the immunostaining pattern for MIF in a Dukes C colon cancer associated with a good prognosis (more than 8 years of survival postdiagnosis) and in a Dukes C colon cancer associated with a dismal prognosis (1 year of survival postdiagnosis), respectively. Panels G-I also illustrated different tissue areas selected for immunohistochemical analysis (in G: epithelial tumor tissue area, in H-I: adjacent peritumoral connective tissue). 
formed by means of Western blotting, Fig. 1, C to E illustrates the check on the presence of galectin-3 and MIF mRNAs, and cDNA quality in the four human colon cancers and the two cell lines used as positive controls.

Fig. 1, F-G shows the immunostaining pattern for galectin-3 in a Dukes A colon cancer associated with a favorable prognosis (more than 8 years of survival postdiagnosis) and in a Dukes B colon cancer associated with a dismal prognosis (2 years of survival postdiagnosis), respectively. In the same way, Fig. 1, H-I shows the immunostaining pattern for MIF in a Dukes C colon cancer associated with a good prognosis (more than 8 years of survival postdiagnosis) and in a Dukes $C$ colon cancer associated with a dismal prognosis (1 year of survival postdiagnosis), respectively.

MIF and Galectin-3 Expression in Normal Colon Tissue, Primary Colorectal Tumors, and Hepatic Metastases from Colorectal Cancers

All the significant variations observed in terms of MIF and galectin-3 expression across the groups of samples analyzed in the Results section concerned the immunostaining intensity in immunopositive tissue areas, which was quantified by means of the Mean Optical Density variable (MOD). In contrast, the Labeling Index (LI, i.e., the percentage of immunopositive tissue area) of the MIF and galectin-3 expression was uniformly high and did not vary significantly across the groups of samples. This variable thus will not be taken into consideration in the data reported in the Results section. (In the following, the terms "galectin-3-or MIF-expression" thus mean "immunostaining intensity.")

With respect to the epithelial tissue (Fig. 2A), the levels of expression in terms of staining intensity (the MOD variable) of both the MIF $(P=0.0009)$ and the galectin-3 ( $P=0.00003)$ significantly increased both in the tumors and the metastases (with less significant p-values because of the smaller number of cases in the latter tissue group), as compared with the normal samples. With respect to the connective tissue (Fig. 2B), a slight increase in the level of galectin-3 expression was observed in the tumors $(P=0.03)$ while the metastases exhibited a decrease in both galectin-3 $(P=0.01)$ and MIF expression $(P=0.04)$, when compared with the normal samples. A comparison of Fig. 2A and B shows that, in each category of specimens (i.e., normal, tumor or metastasis), compared with its connective counterpart, epithelial tissue exhibited much higher levels of MIF and galectin-3 expression. Wilcoxon tests (for paired samples) confirmed these observations $(P<0.001$ for normal samples, $P$ $<10^{-6}$ for tumor samples and $P=0.005$ for metastases). We also investigated whether the tumor location (rectum versus colon) influenced galectin-3 and MIF expression. This analysis was performed on the samples classified as Dukes A or B only because these included a large majority of the rectal tumors analyzed (i.e., 15 cases of 19). The results evidenced no significant variations between rectal and colon tumors for the different markers and tissues investigated (data not shown). Finally, a survival analysis confirmed that the survival periods did not significantly vary when patients suffering of (Dukes A and B) rectal tumors were compared with those with (Dukes A and B) colon tumors (data not shown). This is why rectal and colon tumors were not distinguished afterward.

\section{Correlation between MIF and Galectin-3 Expression (Staining Intensity) in Colorectal Tumor Specimens}

Fig. 2, C-D shows the levels of galectin-3 expression (vertical axis) as function of the levels of MIF expression (horizontal axis) measured in epithelial and connective tumor tissue, respectively. Kendall rank correlation tests showed that while no statistically significant correlation could be evidenced between MIF and galectin-3 staining intensities measured in epithelial tissue (Fig. 2C), a positive and significant correlation (Kendal tau $=0.28, P=$ 0.001) was present between these two staining intensities in connective tissue (Fig. 2D). Furthermore, we established that the cases that contributed the most to this correlation were those associated with short survival periods (i.e., fewer than 5 years, all the Dukes stages taken together). Indeed, whereas the cases associated with long survival periods (i.e., exceeding 6 years) did not exhibit any significant correlation (Fig. 2E), those associated with short survival periods (Fig. 2F) revealed a clearly positive correlation (Kendal tau $=0.49, P=$ 0.0002).

\section{Prognostic Value of MIF and Galectin-3 Staining Intensities}

To investigate whether prognostic groups could be determined, we analyzed the distribution of the patients' survival periods as function of the levels of galectin-3 and MIF expression in epithelial and connective tissue (Fig. 3). We separately analyzed the tumors classified as Dukes A or B and those classified as Dukes C or D. In Fig. 3, the living patients are represented as open dots and the dead ones as black dots; the vertical lines establish two thresholds, which enabled prognostic groups to be determined as demonstrated below. These thresholds were determined (by means of a decision tree technique) to discriminate at best the dead patients from the patients associated with a long survival 
Epithelial Tissue

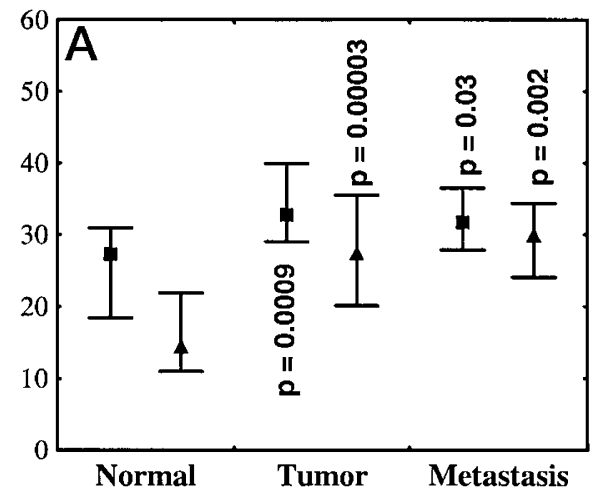

Epithelial Tissue

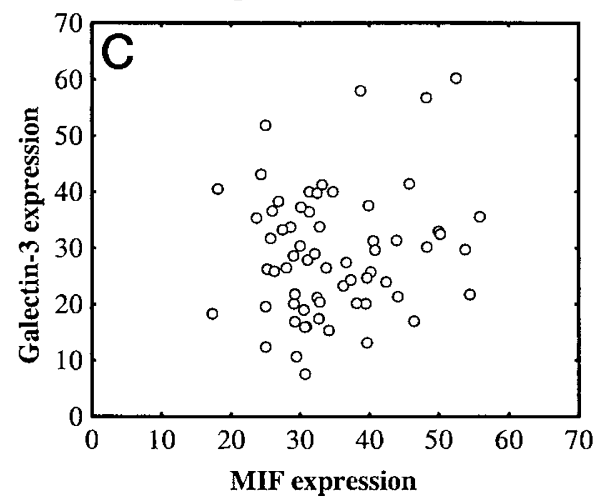

Connective Tissue Patients with Long Survival Periods

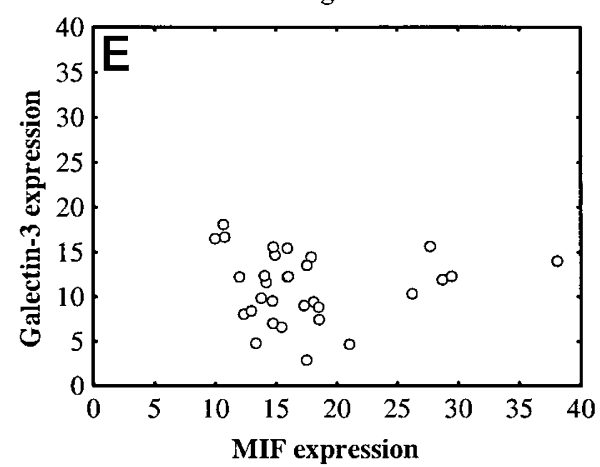

Connective Tissue

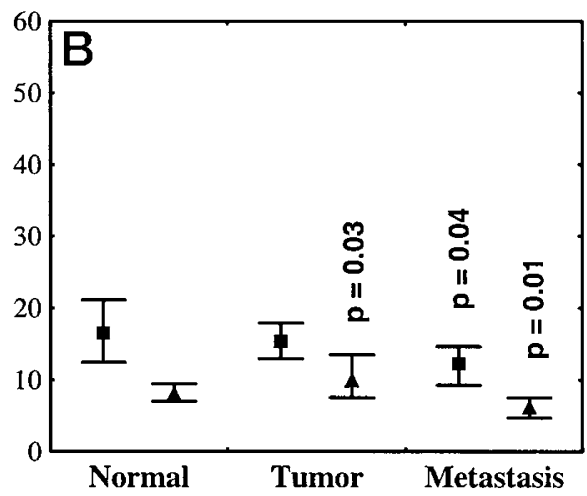

Connective tissue

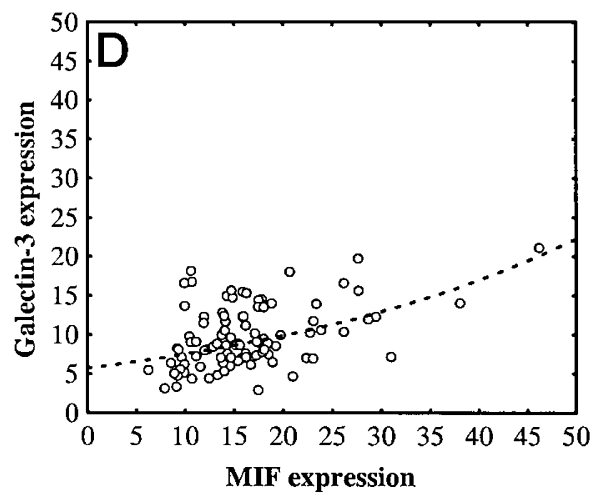

Connective Tissue

Patients with Short Survival Periods

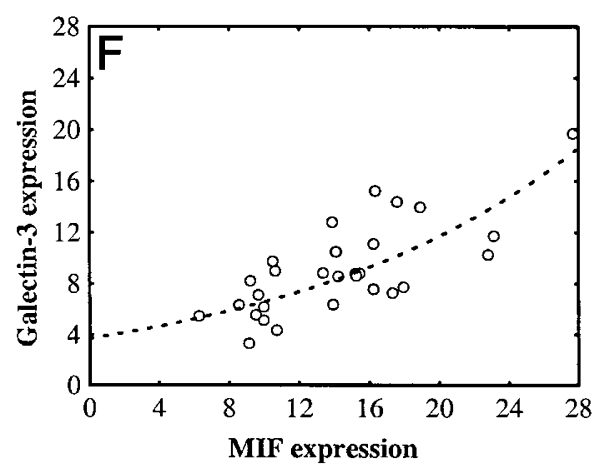

FIGURE 2. Illustration of the variations and correlations of MIF and galectin-3 expression concerning the Mean Optical Density variable (quantifying the immunostaining intensity in arbitrary units). Frames A-B report the variations observed in the epithelial (A) and the connective (B) compartments between normal, tumor and metastasis specimens annotated by the significant p-values (Mann-Whitney tests taking the expression levels in the normal samples as references). The MIF (squares) and galectin-3 (triangles) data are reported as medians (squares or triangles) and the $25 \%$ and $75 \%$ percentiles (bars). Frames C-F illustrate the distribution of galectin-3 expression levels (Y-axis) as function of the MIF expression levels (X-axis) in the tumor samples. While frame $\mathrm{C}$ shows the expression levels measured in each tumor epithelium, frame D reports those measured in each connective tumor tissue. Frames E-F detail frame D data by representing the cases associated with long survival periods (more than 6 years; frame E), and those with short survival periods ( less than 5 years; frame F). The dashed curves indicate a significant correlation between the two variables (detailed in the text)

period (see Materials and Methods). In the case of the Dukes A or B tumors we evidenced a prognostic value for the level of galectin-3 expression in epithelial tissue (Fig. 3A). In contrast, no prognostic information was found for either galectin-3 expression in connective tissue (Fig. 3B), or MIF expression (in both epithelial and connective tissue, data not shown). Inversely, in the cases of the Dukes $\mathrm{C}$ or D tumors a prognostic value was associated with MIF expression in connective tissue (Fig. 3D) but not with MIF expression in epithelial tissue (Fig. 3C) or galectin-3 expression (data not shown).

Four prognostic groups were so determined and were labeled I, II, III and IV, as shown in Fig. 3A and 

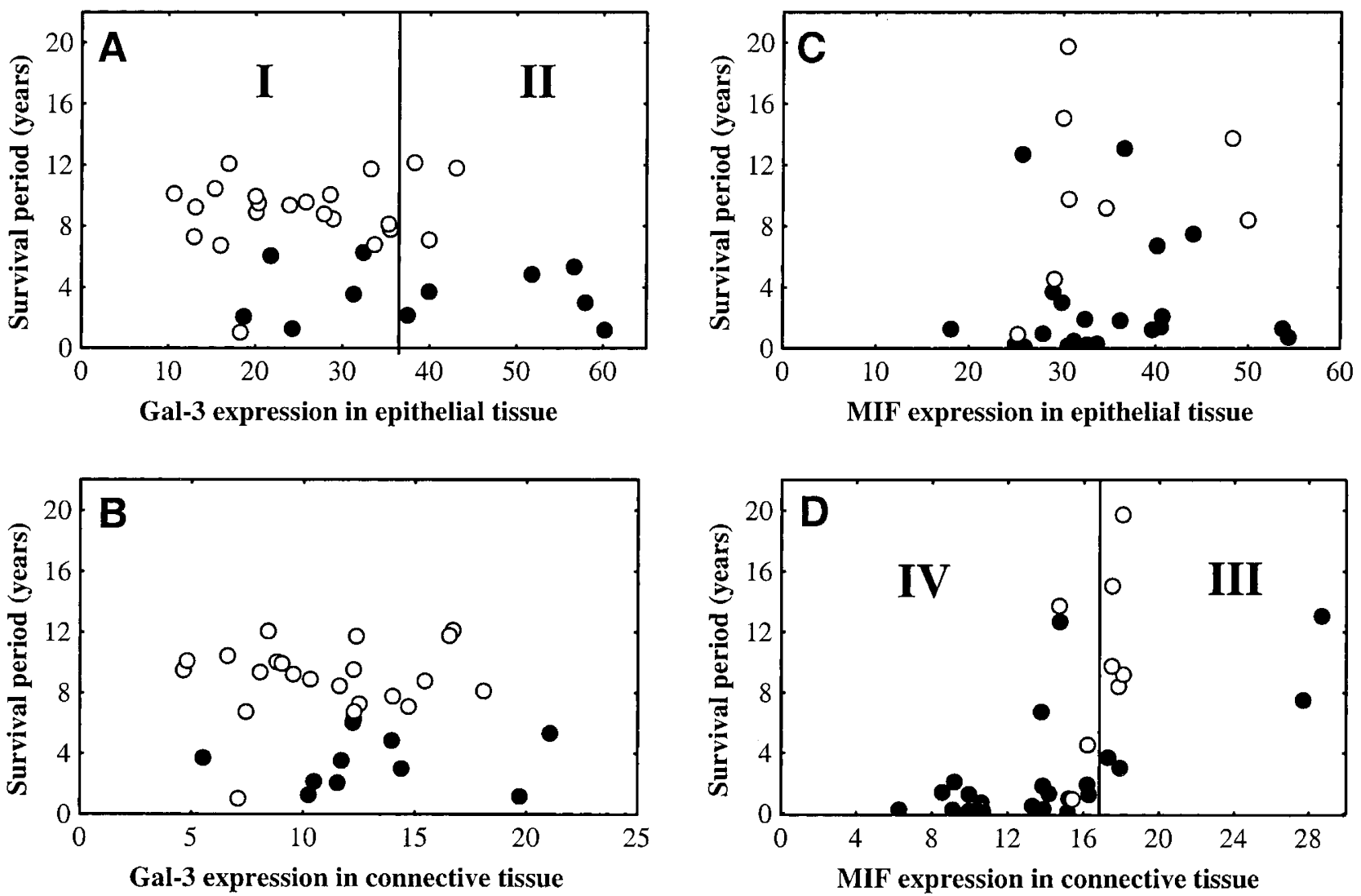

FIGURE 3. Distribution of the patients' survival periods as function either of the galectin-3 (frames A-B) or MIF (frames C-D) expression levels in the epithelial (A-C) and the connective (B-D) compartments. While frames A and B deal with patients suffering from Dukes A or B tumors, frames C and D show patients suffering from Dukes $\mathrm{C}$ or D tumors. The living patients are represented by open dots and the dead ones by black dots. In frames A and D the vertical lines (each of which defines a threshold value on the X-axis) define four new groups of patients as indicated: groups I and II in frame A (i.e., from among the patients with tumors classified as Dukes A or B) and groups III and IV in frame D (i.e., from among the patients with tumors classified as Dukes C or D).

D. While groups I and III included a majority of patients with long survival periods, a majority of patients with short survival periods belonged to groups II and IV. The prognostic value associated with each of these new four groups was demonstrated by means of standard survival time analyses. The results of these analyses are reported in Fig. 4. While Fig. 4A gives the survival curves associated with Dukes A and Dukes B tumors, Fig. 4B shows similar curves obtained when the two new groups I and II (defined as detailed in Fig. 3E) were considered. As is illustrated in Fig. 4B, this new classification enabled a majority of the patients suffering from Dukes B tumors and associated with long survival periods to be allocated to the new group I characterized by very good prognoses (nearly $80 \%$ of survivors after 10 years). As a consequence the patients remaining in the new group II were associated with bad prognoses (below $50 \%$ of survivors after 5 years. The levels of MIF expression (measured in epithelial and connective tumor tissue) exhibited no significant variations between groups I and II (data not shown). Similarly, while Fig. 4C shows the survival curves associated with Dukes C and stage D tumors, Fig. 4D displays similar curves obtained when the two new groups III and IV (defined as detailed in Fig. 3D) are considered. This time, this new classification allocates to new group IV the patients suffering of Dukes C tumors and associated with short survival periods. This group IV is characterized by a generally very bad prognosis (below $20 \%$ of survivors after 5 years, see Fig. 4D). The levels of galectin-3 expression (measured in epithelial and connective tumor tissue) showed no significant variations between groups III and IV (data not shown).

Finally, Fig. 5 summarizes the complete set of prognostic data resulting from the new classification into groups I, II, III and IV (Fig. 5B) in comparison with the data associated with the standard Dukes classification (with an additional stage D, see Fig. 5A). Fig. 5, C-D details the case distribution when crossing the new prognostic groups (I, II, III and IV) and the clinical groups resulting from the 

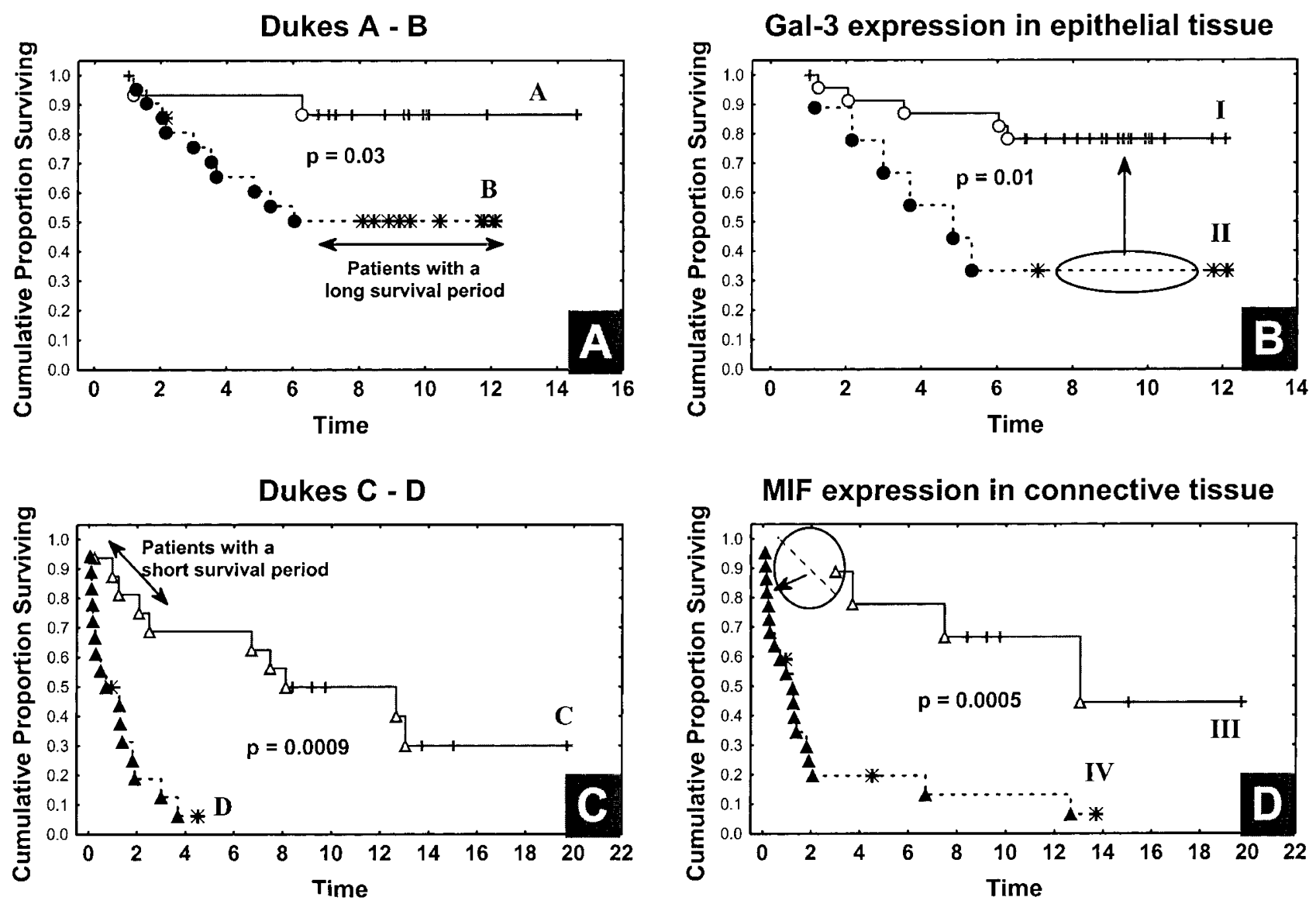

FIGURE 4. Illustration of the survival curves (expressed in years) associated with the Dukes classification (frames A and C) and the new prognostic groups (frames B and D) defined as indicated in Figs. 3A-D. The dead patients are indicated by means of symbols and the living ones by crosses $(+$ ) or stars $\left({ }^{*}\right)$. The p-values reported are computed by means of a standard log-rank test. Frames A-B show that the new distribution of Dukes A and B cases in terms of the new groups I and II enabled the Dukes B cases associated with long survival periods to be allocated to the group I associated with favorable prognoses. Frames C-D show that the new distribution of Dukes C and D cases in terms of the new groups III and IV enabled to allocate the Dukes C cases associated with short survival periods to the group IV associated with very bad prognoses.

Dukes' classification combined with the survival data (i.e., survival periods shorter than 5 years and longer than 6 years). It will be noted that as in Fig. 2, Fig. 5, C-D does not take account of the cases corresponding to living patients with survival periods of fewer than 6 years. This shows that the Dukes B curve (which concerns patients who did not receive chemotherapy) is similar to the Dukes C curve (which concerns patients who did). In contrast, the new group II, which encompasses patients suffering from Dukes A or B tumors (see Fig. 5C) who did not receive chemotherapy, is characterized by a survival curve that is worse than the curve for the new group III, which includes patients suffering from Dukes C or D tumors (see Fig. 5D) who did receive chemotherapy.

Combination of the Prognostic Values Provided by Galectin and MIF Expression in Addition to Standard Clinical Features

Cox regression analyses were carried out to determine the relationship between the survival times and the different variables identified above as po- tentially informative, i.e., the galectin and MIF mean optical densities (MOD) measured in epithelial and connective tissue, as well as the patients' ages, the Dukes tumor stages and the tumor location, giving a total of 7 variables (see Table 1). Table 1 summarizes the best models generated by considering either all the tumor cases together (Models A-B), Dukes A-B tumors (Model C) or Dukes C-D tumors (Models D-E). Each model in Table 1 is characterized by its level of significance, the list of the variables selected, their respective coefficients in the models (in logarithmic and exponential form, respectively) and the corresponding p-values evaluating the significance of the predictive contribution of the variables to the overall model. Model A (taking all the case into account) show that of the 7 variables considered, the most contributive variables to the prediction of the survival times concerned galectin-3 expression (in epithelium and, in a lesser degree, connective tissue). Of the clinical variables, only the patients' ages significantly contributed to the model. Model B presents the best model that we evidenced on the complete series of 


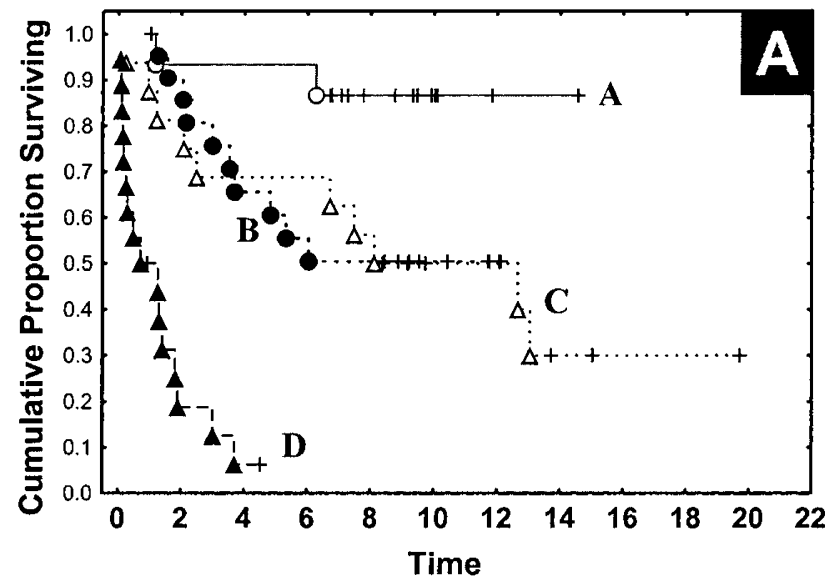

\begin{tabular}{|c|cc|cc|}
\hline C & \multicolumn{2}{|c|}{ Dukes A } & \multicolumn{2}{c|}{ Dukes B } \\
\hline & S & L & S & L \\
\hline I & 0 & 10 & 2 & 9 \\
\hdashline II & 1 & 1 & 4 & 2 \\
\hline
\end{tabular}

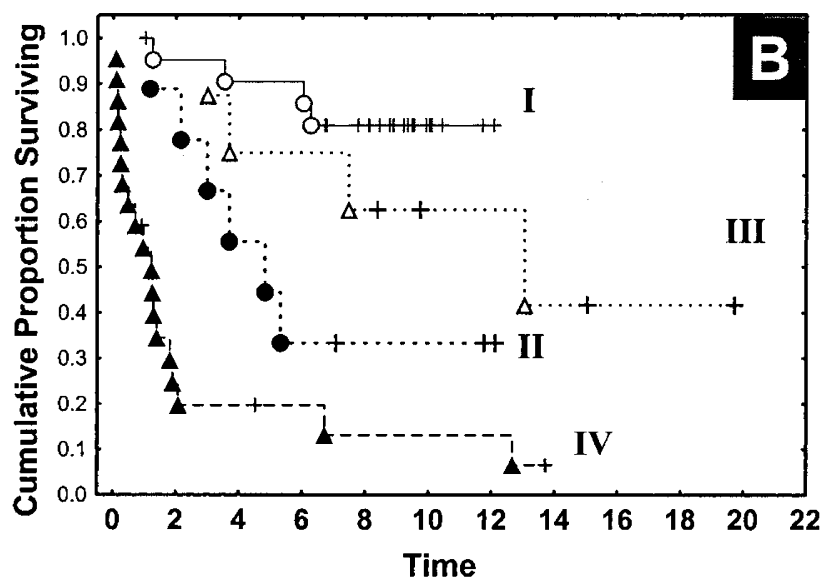

\begin{tabular}{|c|cc:c|}
\hline D & \multicolumn{2}{|c|}{ Dukes C } & Dukes D \\
& S & L & (S) \\
\hline III & 0 & 6 & 2 \\
\hdashline IV & 4 & 3 & 14 \\
\hline
\end{tabular}

FIGURE 5. Illustration of the survival curves (expressed in years) associated with the Dukes classification (frame A) and the new prognostic groups I, II, III, and IV (frame B) defined as indicated in Figs. 3A-D. Frames C-D detail the composition of these prognostic groups in terms of the clinical groups defined on the basis of the Dukes classification and the patients' survival periods. $\mathrm{S}=$ short period ( less than 5 years) and $\mathrm{L}=$ long period ( more than 6 years). In contrast to the survival curves, these latter two tables do not take into account the living patients with survival periods of fewer than 6 years.

tumor cases. This confirms that galectin-3 expression provided highly significant contributions to the predictive model.

Model $\mathrm{C}$ also confirms that the most contributive variable concerned galectin-3 expression in epithelial tissue in the specific case of tumors classified as Dukes A or B. In contrast, when Dukes C or D cases were specifically considered Model D indicated that the Dukes stages (i.e., the difference between Dukes $\mathrm{C}$ and $\mathrm{D}$ stages) was the most contributive variable. This was not surprising as shown in Fig. 4C. However, the best model that we generated to predict the survival times of patients suffering from Dukes $\mathrm{C}$ or D tumors (detailed in Model E) revealed that MIF expression in connective tissue was able to add significant prognostic information to the difference between the tumor stages.

\section{DISCUSSION}

Galectin-3 is expressed in almost all types of epithelia, chondrocytes and in various inflammatory cells (20-24), and the present study shows that the prognostic value that we delineated for galectin-3 concerns the epithelial component of colorectal tumors rather than the connective tissue. In contrast, galectin- 1 is primarily expressed in the stromal cells of human colorectal tissue, with the frequency of strong stromal galectin-1 expression increasing in the normal-adenoma-carcinoma progression (31, 48). In the present study we observed a slight increase in the level of galectin-3 expression (in terms of immunostaining intensity) in the connective tissues of colorectal tumors, whereas the metastases showed a decrease in galectin-3 expression when compared with the normal samples (Fig. 2B).

The prognostic value associated in the present study to galectin-3 expression in epithelial tissue of human colorectal cancers concerns specimens associated with low clinical stages (Dukes A and B; see Fig. 3A). In these specimens we observed that higher levels of galectin-3 expression were associated with shorter periods of survival (Fig. 4B). In other words, these data mean that increased levels 
of galectin-3 are associated with bad prognoses in human colorectal cancers with low clinical stages (Dukes A and B), but no longer in those with higher clinical stages (Dukes C and D). These data therefore suggest that modifications to galectin-3 expression concern the early steps of colorectal cancer progression and no longer the later steps, in which MIF seems to play a significant role, as explained below. These different results were confirmed by means of multivariate analyses taking into account standard clinical variables (Table 1). These analyses also enhanced a strong prognostic contribution for galectin-3 expression when all cases were considered (Table 1).

Our data on galectin-3 partly corroborate those of Sanjuan et al. (48), who observed that patients with stage II and III colorectal cancers whose tumors exhibited strong galectin-3 expression had worse overall and short relapse-free survival periods than those with low galectin-3 expression. Sanjuan et al. (48) also observed that galectin-3 expression is down-regulated in the initial stages of neoplastic progression. We did not observe any such feature but, as discussed below, the term "galectin-3 expression" is very ambiguous: does it refer to the

TABLE 1. Cox Regression Models

\begin{tabular}{|c|c|c|c|c|}
\hline Model/P-Value & Variable & $\beta$ & $\exp (\beta)$ & $P$-value \\
\hline \multicolumn{5}{|l|}{ In all cases } \\
\hline \multirow[t]{7}{*}{ A) $P=0.009$} & Dukes (A-D) & 0.111 & 1.118 & 0.5 \\
\hline & Tumor Location & -0.401 & 0.670 & 0.4 \\
\hline & Patient's age & 0.053 & 1.055 & 0.02 \\
\hline & Gal3-Epith & 0.065 & 1.068 & 0.001 \\
\hline & Gal3-Conn & -0.154 & 0.857 & 0.01 \\
\hline & MIF-Epith & 0.008 & 1.008 & 0.7 \\
\hline & MIF-Conn & -0.005 & 0.995 & 0.9 \\
\hline \multirow[t]{3}{*}{ B) $P=0.0006$} & Patient age & 0.052 & 1.053 & 0.01 \\
\hline & Gal3-Epith & 0.065 & 1.067 & 0.0004 \\
\hline & Gal3-Conn & -0.162 & 0.851 & 0.002 \\
\hline \multicolumn{5}{|c|}{ In Dukes A and B tumors } \\
\hline \multirow[t]{6}{*}{ C) $P=0.002$} & Dukes (A-B) & 1.026 & 2.790 & 0.07 \\
\hline & Patient's age & 0.084 & 1.088 & 0.2 \\
\hline & Gal3-Epith & 0.137 & 1.147 & 0.001 \\
\hline & Gal3-Conn & -0.123 & 0.884 & 0.4 \\
\hline & MIF-Epith & 0.181 & 1.198 & 0.04 \\
\hline & MIF-Conn & -0.094 & 0.910 & 0.2 \\
\hline \multicolumn{5}{|c|}{ In Dukes $C$ and D tumors } \\
\hline \multirow[t]{6}{*}{ D) $P=0.001$} & Dukes (C-D) & 0.836 & 2.307 & 0.008 \\
\hline & Patient's age & 0.051 & 1.052 & 0.1 \\
\hline & Gal3-Epith & 0.031 & 1.092 & 0.4 \\
\hline & Gal3-Conn & 0.014 & 1.014 & 0.9 \\
\hline & MIF-Epith & -0.020 & 0.980 & 0.4 \\
\hline & MIF-Conn & -0.132 & 0.876 & 0.1 \\
\hline \multirow[t]{3}{*}{ E) $P=0.00007$} & Dukes (C-D) & 0.869 & 2.385 & 0.003 \\
\hline & Patient's age & 0.033 & 1.033 & 0.1 \\
\hline & MIF-Conn & -0.145 & 0.865 & 0.03 \\
\hline
\end{tabular}

The "Model $/ P$ value" indicates the overall level of significance of the model. The variables related to MIF and galectin-3 expression are the mean optical densities measured either in tumor epithelium ("Epith") or in peritumoal connective tissue ("Conn"). The equation at the basis of the Cox Regression model is an exponential function of a linear combination of the variables considered, where $\beta$ indicates the coefficient of each variable in the linear combination, and $\exp (\beta)$ its exponential value. The $P$-value indicates the level of significance of the contribution of each variable to the model (so enabling to be concluded that $\beta$ is significantly different from zero). number of cells with galectin-3 expression (corresponding to the LI variable), to the galectin-3 staining intensity per immunopositive cell (corresponding to the MOD variable) or to both? The fact, already emphasized by Itzkowitz (28), remains valid that the data published in the literature on the diagnostic and/or prognostic value of galectin-3 expression in human colorectal carcinomas are very inconsistent. Indeed, as already mentioned in the Introduction, Lotz et al. (29) and Castronovo et al. (30) report decreasing levels of galectin-3 expression in colon carcinomas as compared with normal colon tissue while Irimura et al. (31), Schoeppner et al. (32) and Nakamura et al. (33) report the opposite. The data from our study can be reconciled with those of the second group of authors because we also observed increasing levels of galectin-3 (staining intensity) in colorectal tumors as compared with normal colon tissue. In the mentioned editorial, Itzkowitz (28) reports that the most comprehensive of the above-mentioned studies was the one published by Schoeppner et al. (32), who showed weak or no galectin-3 expression in normal colonic mucosa remote from colon cancers, with the expression progressively increasing in colonic adenomas, colon cancers at more advanced stages, and colon cancer metastases as compared with their primary tumors. While increased galectin-3 expression in colorectal cancers was associated with lower survival rates, this effect was not found to be independent of, or dependent on, tumor stage (32). Our study clearly indicates that this effect is dependent on the clinical stages.

It has been shown recently that circulating galectin-3 might indicate tumor progression (and not only of colorectal origin) because the galectin-3 concentration in sera from patients with metastatic pathologies is higher than in sera from patients with localized tumors (49). However, because only 25 patients with gastrointestinal cancer were included and the source of the serum galectin-3 is unclear, further studies are warranted to delineate a correlation between the metastasizing phenotype and this serum parameter. Itzkowitz (28) asserts that possible explanations for the varying conclusions in the mentioned studies published in the literature on the diagnostic and/or prognostic values of galectin-3 expression include differences in sample size and selection, different methodological approaches, the use of different antibodies and differences in the interpretation of staining. The present study clearly indicates that the significant variations observed in terms of MIF and galectin-3 expression at protein level across the different groups analyzed in the Results section were connected with immunostaining intensity quantified by means of the Mean Optical Density variable (MOD, see Materials and Methods). In contrast, the 
Labeling Index (the LI variable that refers to the percentage of immunopositive cells) for the MIF and galectin-3 expression did not significantly vary across the different groups. Thus, in our study the MIF and galectin-3 staining intensities related to significant prognostic values, while the percentages of cells immunopositive for these proteins did not.

The present study strengthens the case that galectin-3 expression is associated with colorectal cancer development. In principle, galectin-3 can interfere with tumor progression at different levels. Galectin-3 can for example maintain tumor growth by decreasing cell loss via apoptosis, although it is emerging that the profile of galectin-3 activity critically depends on the cell type $(50,51)$. Galectin- 3 may also be a critical determinant for the anchorageindependent survival of disseminating cancer cells in the circulation during metastases (51). Galectin-3 can also significantly modify the metastatic capacities of colorectal cancer cells: using lectin blocking by sugar or antibody or transfected cell lines a marked decrease in liver colonization and the spontaneous metastasis of the sense-transfected colon cancer cells were observed $(52,53)$. Another level where galectin-3 can be functional is in interfering with activities of other galectins, as recently shown for galectin-1 as negative growth regulator (54). Because we have previously documented that it is essential to monitor presence of more than galectin- 1 and -3 in colorectal cancer by delineating galectin- 8 as suppressor activity (43), this aspect will deserve special attention in further studies.

It should be emphasized that data obtained from in vitro with cell lines, whose biological behavior dramatically differs from human clinical samples may not be readily extrapolated to the clinical situation.

MIF was one of the first cytokine activities to be discovered and was originally described as a T lymphocyte product that inhibited the random migration of macrophages $(36,37)$. Similar to other proinflammatory cytokines, it not only modulates immune functions but also has a bearing on angiogenesis and tumor growth, rendering tumorgrowth-promoting activities likely besides an expected role in anti-tumor immunity $(36,37)$. MIF has already been proved to be over-expressed in colon cancers as compared with normal colon tissues (38), and to participate in colon cancer progression (39). Takahashi et al. (55) studying the murine colon cancer line colon 26 substantiated that in this model MIF is profoundly involved in tumor growth stimulation. Elicitors for increase of intracellular MIF presence were suggested to serve as potential targets for therapy, a conclusion extended to B cell lymphoma and melanoma in ensuing studies $(56,57)$. Corroborating these reports and underlining the asserted role of MIF in tumor progression, Takahashi et al. suggested the possibility that MIF promotes tumor cell growth in conjunction with other growth factors. In addition, Ogawa et al. (58) found that MIF activity may be involved in tumorigenesis via the promotion of angiogenesis. Thus, MIF-like other proinflammatory cytokines such as interleukin-6 (for reviews, see 59-61; for recent report on this cytokine as growth factor in the case of prostate cancer, see 62) -is not only involved in immunoregulation but also in tumor cell growth. Our report clearly substantiates that MIF is present in human colorectal cancer tissue in situ and encourages further histopathological investigations targeting MIF presence, an emerging topic with potential therapeutic implications. The prognostic value that we evidenced for MIF concerns the connective tissue of colorectal tumors associated with high clinical stages (Dukes C and D; see Figs. 3D and 4D). These data therefore suggest that MIF detected in the vicinity can influence the biological behavior of highly malignant colorectal epithelial cells. Data on the Kendall correlation tests shown in Fig. 2D and F even intimate a functional cooperation with galectin-3 present in this compartment for patients with short survival periods. Remarkably, sustained growth in cholesteatomas had been correlated by the same statistical procedure to the score of immunopositive cells for these two effectors (34). Emergence of the same correlation in these two tissue types warrants to broaden the data basis on this aspect, keeping in mind the suggestive functional overlap of MIF and galectin-3 in growth regulation and angiogenesis.

In conclusion, the data from the present study show that the survival curve associated with the Dukes B colorectal cancer patients (who did not receive chemotherapy) is similar to the survival curve of the Dukes C colorectal cancer patients (who did receive chemotherapy). In contrast, the new group II that we identified in the present study on the basis of galectin-3 expression in the epithelial tissue of colorectal cancers associated with Dukes A and B clinical stages, includes patients who did not receive chemotherapy. The survival curve associated with this group is worse than the curve characterizing the new group III, which includes patients suffering from a Dukes C or D colorectal tumor (see Fig. 5D) who received chemotherapy. These data therefore enabled us to identify from among the patients suffering from a Dukes A or B colorectal tumor the group II of patients for whom chemotherapy would be recommended in our view. Similarly, the new group IV identified in the present study could be considered as identifying cases requiring more "aggressive" adjuvant therapy than that currently provided. Lastly, our data suggest that the pro-inflammatory MIF cytokine could influence the late stages of colorectal 
cancer development. Immunomodulatory compounds, in particular those affecting MIF presence, could therefore be assayed in addition to standard chemotherapy for patients whose colorectal cancers are associated with a high clinical stage. As suggested from model animal studies on colon carcinoma, lymphoma and melanoma, tumor progression could be reduced by targeting MIF expression. In this respect, our data provide first histopathological evidence in colorectal cancer supporting this emerging concept.

Acknowledgments: We gratefully acknowledge Dr. J.L. Wang for kindly providing the galectin-3 expression vector. This work has been carried out on the basis of grants awarded by the "Amis de l'Institut Bordet" (Brussels, Belgium), the "Fonds de la Recherche Scientifique Médicale" (FRSM, Brussels, Belgium), the "Fondation Yvonne Boël" (Brussels, Belgium) and the Wilhelm Sander-Stiftung (Munich, Germany).

\section{REFERENCES}

1. Wilmink AB. Overview of the epidemiology of colorectal cancer. Dis Colon Rectum 1997;40:483-93.

2. Maghfoor I. Colorectal cancer. The Lancet 1999;353:391-9.

3. Ries LA, Wingo PA, Miller DS, Howe HL, Weir HK, Rosenberg $\mathrm{HM}$, et al. The annual report to the nation on the status of cancer, 1973-1997, with a special section on colorectal cancer. Cancer 2000;88:2398-2424.

4. Cohen AM. Colorectal cancer: evolving concepts in diagnosis, treatment, and prevention. CA Cancer J Clin 1999;49: 199-201.

5. Wils JA. Adjuvant treatment in Dukes' B and C disease. Annu Oncol 2000;11(Suppl):37-43.

6. Lindblom A. Improved tumor staging in colorectal cancer. N Engl J Med 1998;339:264-5.

7. Ratto C, Sofo L, Ippoliti M, Merico M, Doglietto GB, Crucitti F. Prognostic factors in colorectal cancer. Literature review for clinical application. Dis Colon Rectum 1998;41:1033-49.

8. Gennari L, Doci R, Rossetti C. Prognostic factors in colorectal cancer. Hepatogastroenterology 2000;47:310-4.

9. Dukes CE. The classification of cancer of the rectum. J Pathol Bacteriol 1932;35:323-32.

10. Freedman LS, Macaskill P, Smith AN. Multivariate analysis of prognostic factors for operable rectal cancer. Lancet 1984;2: 733-6.

11. Newland RC, Dent OF, Lyttle MN, Chapuis PH, Bokey EL. Pathologic determinants of survival associated with lymph node metastases. Cancer 1994;60:2318-24.

12. Vogelstein B, Fearon ER, Hamilton SR, Kern SE, Preisinger AC, Leppert M, et al. Genetic alterations during colorectaltumor development. N Engl J Med 1988;319:525-32.

13. Jen J, Powell SM, Papadopoulos N, Smith KJ, Hamilton SR, Vogelstein B, et al. Molecular determinants of dysplasia in colorectal lesions. Cancer Res 1994;54:5523-6.

14. Shih IM, Wang TL, Traverso G, Romans K, Hamilton SR, Ben-Sasson S, et al. Top-down morphogenesis of colorectal tumors. Proc Natl Acad Sci USA 2001;98:2640-5.

15. Yogeeswaran G. Cell surface glycolipids and glycoproteins in malignant transformation. Adv Cancer Res 1983;38:289-350.

16. Alhadeff JA. Malignant cell glycoproteins and glycolipids. Crit Rev Oncol Hematol 1989;9:37-107.
17. Hakomori S. Tumor malignancy defined by aberrant glycosylation and sphingo(glyco)lipid metabolism. Cancer Res 1996;56:5309-18.

18. Gabius HJ. Concepts of tumor lectinology. Cancer Invest 1997;15:454-64.

19. Brockhausen I, Schutzbach J, Kuhns W. Glycoproteins and their relationship to human disease. Acta Anat 1998;161:3678.

20. Gabius HJ. Animal lectins. Eur J Biochem 1997;243:543-76.

21. Hirabayashi J (editor). Recent topics on galectins. Trends Glycosci Glycotechnol 1997;9:1-180.

22. Perillo NL, Marcus ME, Baum LG. Galectins: versatile modulators of cell adhesion, cell proliferation, and cell death. J Mol Med 1998;76:402-12.

23. Hughes RC. Secretion of the galectin family of mammalian carbohydrate-binding proteins. Biochim Biophys Acta 1999; 1473:172-85.

24. Sano H, Liu FT. Galectins: another family of chemoattractants? Mod Asp Immunobiol 2001;2:4-6.

25. Ohannesian DW, Lotan D, Thomas P, Jessup JM, Fukuda M, Gabius HJ, et al. Carcinoembryonic antigen and other glycoconjugates act as ligands for galectin-3 in human colon carcinoma cells. Cancer Res 1995;55:2191-9.

26. Lahm H, Andre S, Hoeflich A, Fischer JR, Sordat B, Kaltner H, et al. Comprehensive galectin fingerprinting in a panel of 61 human tumor cell lines by RT-PCR and its implications for diagnostic and therapeutic procedures. J Cancer Res Clin Oncol 2001;127:375-86.

27. Bresalier RS, Byrd JC, Wang L, Raz A. Colon cancer mucin: a new ligand for the $\beta$-galactoside-binding protein galectin-3. Cancer Res 1996;56:4354-7.

28. Itzkowitz SH. Galectins. Multipurpose carbohydrate-binding proteins implicated in tumor biology. Gastroenterology 1997;113:2003-5.

29. Lotz MM, Andrews CW Jr, Korzelius CA, Lee EC, Steele GD Jr, Clarke A, et al. Decreased expression of Mac-2 (carbohydrate binding protein 35) and loss of its nuclear localization are associated with the neoplastic progression of colon carcinoma. Proc Natl Acad Sci USA 1993;90:3466-70.

30. Castronovo V, Campo E, Van Der Brule FA, Claysmith AP, Cioce V, Liu FT, et al. Inverse modulation of steady-state messenger RNA levels of two non-integrin laminin-binding proteins in human colon carcinoma. J Natl Cancer Inst 1992; 84:1161-9.

31. Irimura T, Matsushita Y, Sutton RC, Carralero D, Ohannesian DW, Cleary KR, et al. Increased content of an endogenous lactose-binding lectin in human colorectal carcinoma progressed to metastatic stages. Cancer Res 1991;51:387-93.

32. Schoeppner HL, Raz A, Ho SB, Bresalier RS. Expression of an endogenous galactose-binding lectin correlates with neoplastic progression in the colon. Cancer 1995;75:2818-26.

33. Nakamura $M$, Inufusa $H$, Adachi $T$, Aga $M$, Kurimoto $M$, Nakatani Y, et al. Involvement of galectin-3 expression in colorectal cancer progression and metastasis. Int J Oncol 1999;15:143-8.

34. Choufani G, Ghanooni R, Decaestecker C, Delbrouck K, Simon P, Schuring MP, et al. Detection of macrophage migration inhibitory factor (MIF) in human cholesteatomas and functional implications of correlations to recurrence status and to expression of matrix metalloproteinases-3/9, retinoic acid receptor-beta, and anti-apoptotic galectin-3. Laryngoscope 2001;111:1656-62.

35. Cordon-Cardo C, Prives C. At the crossroads of inflammation and tumorigenesis. J Exp Med 1999;190:1367-70.

36. Mitchell RA, Bucala R. Tumor growth-promoting properties of macrophage migration inhibitory factor (MIF). Semin Cancer Biol 2000;10:359-66. 
37. Nishihira J. Macrophage migration inhibitory factor (MIF): its essential role in the immune system and cell growth. J Interferon Cytokine Res 2000;20:751-62.

38. Shkolnik T, Livni E, Reshef R, Lachter J, Eidelman S. Comparison of two lymphokines (macrophage migration inhibition, leukocyte adherence inhibition factors) and carcinoembryonic antigen in colorectal cancer and colonic premalignant lesions. Am J Gastroenterol 1987;82:1275-8.

39. Reshef R, Livni E, Lachter J, Suprun H, Eidelman S, Shkolnik T. Colon cancer bearing rats produce a lymphokine which induces macrophage migration inhibition (MIF) in vitro. Comp Immunol Microbiol Infect Dis 1988;11:61-9.

40. Moertel CG, Fleming TR, Macdonald JS, Haller DG, Laurie JA, Goodman PJ, et al. Levamisole and fluorouracil for adjuvant therapy of resected colon carcinoma. N Engl J Med 1990;322:352-8

41. Camby I, Belot N, Rorive S, Lefranc F, Maurage CA, Lahm H, et al. Galectins are differentially expressed in supratentorial pilocytic astrocytomas, astrocytomas, anaplastic astrocytomas and glioblastomas, and significantly modulate tumor astrocyte migration. Brain Pathol 2001;11:12-26.

42. Rosengren E, Bucala R, Aman P, Jacobsson L, Odh G, Metz $\mathrm{CN}$, et al. The immunoregulatory mediator macrophage migration inhibitory factor (MIF) catalyzes a tautomerization reaction. Mol Med 1996;2:143-9.

43. Nagy N, Bronckart Y, Camby I, Phillipart P, Lahm H, Kaltner $\mathrm{H}$, et al. Galectin-8 expression decreases in cancer as compared to normal and dysplastic human colon tissue and acts significantly on human colon cancer cell migration. Gut 2002;50:392-401.

44. Belot N, Rorive S, Doyen I, Lefranc F, Bruyneel E, De Decker $\mathrm{R}$, et al. Molecular characterization of cell-substratum attachments in human glial tumors relates to prognostic features. Glia 2001;36:375-90.

45. Hyndman D, Cooper A, Pruzinsky S, Coad D, Mitsuhashi M. Software to determine optimal oligonucleotide sequences based on hybridization simulation data. Biotechniques 1996; 20:1090-7.

46. Decaestecker C, van Velthoven R, Petein M, Janssen T, Salmon I, Pasteels JL, et al. The use of the decision tree technique and image cytometry to characterize aggressiveness in World Health Organization (WHO) grade II superficial transitional cell carcinomas of the bladder. J Pathol 1996;178:274-83.

47. Francois C, Decaestecker C, Petein M, van Ham P, Peltier A, Pasteels JL, et al. Classification strategies for the grading of renal cell carcinomas, based on nuclear morphometry and densitometry. J Pathol 1997;183:141-50.

48. Sanjuan X, Fernandez PL, Castells A, Castronovo V, Van Den Brule F, Liu FT, et al. Differential expression of galectin-3 and galectin-1 in colorectal cancer progression. Gastroenterology 1997;113:1906-15.
49. Iurisci I, Tinari N, Natoli C, Angelucci D, Cianchetti E, Iacobelli S. Concentrations of galectin-3 in the sera of normal controls and cancer patients. Clin Cancer Res 2000;6:138993.

50. Yang RY, Hsu DK, Liu FT. Expression of galectin-3 modulates T-cell growth and apoptosis. Proc Natl Acad Sci USA 1996; 93:6737-42.

51. Kim HR, Lin HM, Biliran H, Raz A. Cell cycle arrest and inhibition of anoikis by galectin-3 in human breast epithelial cells. Cancer Res 1999;59:4148-54.

52. Bresalier RS, Mazurek N, Sternberg LR, Byrd JC, Yunker CK, Nangia-Makker P, et al. Metastasis of human colon cancer is altered by modifying expression of the beta-galactosidebinding protein galectin-3. Gastroenterology 1998;115:28796.

53. Inufusa $H$, Nakamura $M$, Adachi $T$, Aga $M$, Kurimoto $M$, Nakatani Y, et al. Role of galectin-3 in adenocarcinoma liver metastasis. Int J Oncol 2001;19:913-9.

54. Kopitz J, von Reitzenstein C, Andre S, Kaltner H, Uhl J, Ehemann $\mathrm{V}$, et al. Negative regulation of neuroblastoma cell growth by carbohydrate-dependent surface binding of galectin-1 and functional divergence from galectin-3. J Biol Chem 2001;276:35917-23.

55. Takahashi N, Nishihira J, Sato Y, Kondo M, Ogawa H, Ohshima $\mathrm{T}$, et al. Involvement of macrophage migration inhibitory factor (MIF) in the mechanism of tumor cell growth. Mol Med 1998;4:707-14.

56. Chesney J, Metz C, Bacher M, Peng T, Meinhardt A, Bucala R. An essential role for macrophage migration inhibitory factor (MIF) in angiogenesis and the growth of a murine lymphoma. Mol Med 1999;5:181-91.

57. Shimizu T, Abe R, Nakamura H, Ohkawara A, Suzuki M, Nishihira J. High expression of macrophage migration inhibitory factor in human melanoma cells and its role in tumor cell growth and angiogenesis. Biochem Biophys Res Commun 1999;264:751-8.

58. Ogawa H, Nishihira J, Sato Y, Kondo M, Takahashi N, Oshima T, et al. An antibody for macrophage migration inhibitory factor suppresses tumor growth and inhibits tumorassociated angiogenesis. Cytokine 2000;12:309-14.

59. Sogn JA. Tumor immunology: the glass is half full. Immunity 1998;9:757-63.

60. Gabius HJ. Probing, the cons and pros of lectin-induced immunomodulation: case studies for the mistletoe lectin and galectin-1. Biochimie 2001;83:659-66.

61. Smith PC, Hobisch A, Lin DL, Culig Z, Keller ET. Interleukin-6 and prostate cancer progression. Cytokine Growth Factor Rev 2001;12:33-40.

62. Giri D, Ozen M, Ittmann M. Interleukin-6 is an autocrine growth factor in human prostate cancer. Am J Pathol 2001; 159:2159-65. 